\title{
Teorías subjetivas de docentes directivos sobre factores que definen la eficacia de los planes de mejoramiento educativo ley SEP
}

\author{
Subjective theories by educational directors on factors that define \\ the effectiveness of educational improvement plans in SEP-law \\ Teorias subjetivas de diretores sobre fatores que definem a eficácia \\ dos projetos de melhoramento educacional Lei SEP \\ César Rivera Núñez, ${ }^{a}$ Paulo Volante Beach. ${ }^{b}$ \\ a Departamento Psicología, Universidad Santo Tomás, Chile. Fono: 56-9-88520877. \\ Correo electrónico: cesar.rivera.nu@gmail.com \\ b Facultad de Educación, Pontificia Universidad Católica, Chile. Fono: 56-2-22354-5377. \\ Correo electrónico: pvolante@puc.cl
}

\begin{abstract}
RESUMEN
El objetivo del estudio de tipo cualitativo fue conocer las teorías subjetivas sobre factores que definen la eficacia de los Planes de Mejoramiento Educativo - Ley SEP desde la perspectiva de docentes directivos y contrastar estos factores con mediciones SIMCE. Se efectuaron diez entrevistas semiestructuradas y revisión de los planes de mejoramiento elaborados por las escuelas. El análisis de datos se realizó mediante el programa Atlas-ti, basándose en los procesos de codificación según los criterios de la Grounded Theory. Los resultados revelaron cinco tipos de factores vinculados a la eficacia, que se centraban principalmente en aspectos curriculares, de estructuración y organización, factores personales, profesionales y de convivencia escolar, los que se sustentan en la existencia de recursos suficientes y adecuados. Respecto a la vinculación de éstos con resultados SIMCE se observaron leves diferencias entre los establecimientos estudiados.
\end{abstract}

Palabras clave: plan de mejoramiento educativo, eficacia, teorías subjetivas, Ley de Subvención Escolar Preferencial.

ABSTRACT
The aim of this qualitative study was to determine the subjective theories related to factors that define the effectiveness of Educational Improvement Plans - SEP law from the perspective of educational directors, and then to contrast these factors with SIMCE measurements. Ten semi-structured interviews were carried out and Improvement Plans developed by schools were reviewed. The data analysis was performed using the Atlas-ti software, based on the three coding processes according to the criteria of Grounded Theory. The results revealed the existence of five types of factors related to the effectiveness: focused primarily on curricular aspects of structuring and organization, personal factors, school life and professional factors, that are based on the existence of sufficient and appropriate resources. Regarding these factors link to SIMCE results, were observed slight differences between the studied facilities.

Key words: educational improvement plan, effectiveness, subjective theories, Preferential School Subvention Law.

\section{RESUMO}

O objetivo do estudo qualitativo foi conhecer as teorias subjetivas sobre os fatores que definem a eficácia dos Planejamentos de Melhoria Educacional - Lei SEP a partir da perspectiva de diretores e contrastar esses 
Estudios Pedagógicos XLI, N 1: 237-256, 2015

TEORÍAS SUBJETIVAS DE DOCENTES DIRECTIVOS SOBRE FACTORES QUE DEFINEN LA EFICACIA DE LOS PLANES DE MEJORAMIENTO EDUCATIVO LEY SEP

fatores com medições SIMCE. Foram realizadas dez entrevistas semiestruturadas e feita revisão dos projetos de melhoramento elaborados pelas escolas. A análise dos dados foi realizada por meio do programa Atlas-ti, com base nos processos de codificação de acordo com os critérios da Grounded Theory. Os resultados revelaram a existência de cinco tipos de fatores relacionados à eficácia, concentrando-se principalmente nos aspectos curriculares, de estruturação e organização, fatores pessoais, profissionais e de convivência escolar, os quais se sustentam na existência de recursos suficientes e adequados. No que diz respeito à ligação de estes com os resultados SIMCE, foram observadas pequenas diferenças entre as instituições estudadas.

Palavras chave: projeto de melhoramento educacional, eficácia, teorias subjetivas, Lei de Subvenção Escolar Preferencial.

\section{INTRODUCCIÓN}

En Chile, en los últimos veinte años, ha existido una serie de programas o planes orientados al mejoramiento de la educación, tanto en lo que respecta a su calidad como a sus resultados. En este sentido, si nos remitimos a la historia reciente, podemos nombrar múltiples iniciativas entre las cuales destaca: el programa P900 que se inició en 1990 y trabajó sobre 900 escuelas vulnerables del país; el proyecto Montegrande introducido durante el año 1997 que benefició la innovación curricular a nivel de educación media, y; el Sistema de Aseguramiento de la Calidad de la Gestión Escolar (SACGE) que a partir del 2005 (González, 2012) formó la base para los actuales parámetros educativos.

Es en este contexto que, a partir del año 2008, son iniciados los primeros planes de mejoramiento educativo creados y financiados bajo el alero de la denominada Ley $\mathrm{N}^{\circ}$ 20.248 de Subvención Escolar Preferencial (Ley SEP), generando una serie de cambios en la forma de ver y hacer educación en nuestro país. Recordemos que esta ley entrega recursos económicos a los establecimientos educacionales por cada alumno denominado "prioritario", y para hacerse efectiva el sostenedor debe firmar un acuerdo conocido como "Convenio de Igualdad de Oportunidades". Todo lo anterior busca mejorar los aprendizajes de niños y niñas, los cuales son medidos anualmente por el Sistema de Medición de la Calidad de la Enseñanza (SIMCE), constituyéndose en un indicador claro de la eficacia y la efectividad de los planes de mejora, que son, en simples palabras, acciones orientadas a cumplir las metas institucionales e invertir los recursos recibidos (MINEDUC, 2012).

Considerando lo anterior, así como las modificaciones incorporadas a la Ley SEP durante el 2011, ésta toma progresivamente más relevancia. En primer lugar, se constata una gran cantidad de colegios adscritos al convenio, que en la actualidad suman cerca de 7.500, lo que representa una cobertura superior al $80 \%$ del total de establecimientos educacionales que cumplen las condiciones para ser beneficiados por esta ley (MINEDUC, 2012); en segundo lugar, otro factor a tener en cuenta es la gran cantidad de recursos invertidos anualmente, cuyo aumento en los establecimientos beneficiarios se calcula en torno al 60\% (Bellei, 2010); en tercer lugar, la ley contempla a contar del 2013 el ingreso de la educación media, y; en cuarto lugar, pero no menos importante, se observa un crecimiento considerable de las denominadas Asistencias Técnicas Educativas (ATE), las cuales desde finales del 2007 hasta 2011 aumentaron desde menos de 100 a casi 500 oferentes, ampliando además su oferta de servicios.

Es por esto que estudiar la eficacia de los Planes de Mejoramiento Educativo (PME) se hace necesario principalmente desde la visión de los docentes directivos, ya que son ellos, con sus representaciones mentales y/o teorías subjetivas, quienes, en la mayoría de 
los casos, figuran como los principales responsables de la creación, ejecución, supervisión y monitoreo de los PME, además de estar a cargo de buena parte de las decisiones que toma la entidad educativa -por lo menos a nivel municipal- en relación tanto con el logro de aprendizajes y su mejoramiento, como de la administración y la gestión del establecimiento en su diario acontecer. En este sentido, los docentes directivos se convierten en actores clave para definir y descubrir qué se espera de un plan de mejora y cómo se consideraría la eficacia del mismo, pues es en base a sus representaciones y a cómo ellos estructuran las demandas emanadas desde el estado, que los planes se conforman y desarrollan dentro de los establecimientos, con focos y características que los diferencian a pesar de la existencia de un marco legal común.

De esta forma, en esta investigación se trabajaron las siguientes preguntas: ¿cuáles son las teorías que poseen los docentes directivos sobre los factores que definen la eficacia de los Planes de Mejoramiento Educativo?, y ¿cómo se vinculan estos factores con indicadores gubernamentales relacionados con la eficacia de los Planes de Mejoramiento Educativo? A partir de estas interrogantes se plantearon los siguientes objetivos generales: a) "conocer las teorías subjetivas sobre factores que definen la eficacia de los Planes de Mejoramiento Educativo - Ley SEP desde la perspectiva de docentes directivos de los Establecimientos Municipalizados de la comuna de Vicuña”, y b) "contrastar los factores identificados por los directivos de la comuna de Vicuña con indicadores gubernamentales asociados con la eficacia de los Planes de Mejoramiento Educativo". Para lograr estos objetivos: 1) se analizaron las teorías relacionadas con la definición de los PME-SEP, 2) se identificaron los factores que los docentes vincularon con su eficacia, y 3) se contrastaron estas teorías con las últimas mediciones SIMCE que obtuvieron estos establecimientos.

\section{MARCO CONCEPTUAL}

\subsection{TEORÍAS SUBJETIVAS}

Este estudio se centró en las teorías subjetivas (TS), pues como menciona Catalán (2004) citando a Clark y Peterson (1990), las teorías y las creencias son una de las tres direcciones básicas del estudio del pensamiento del profesor. En este sentido, consideramos las teorías subjetivas como un tipo particular de representaciones de carácter individual o colectivo (Catalán, 2010), constructos que, en suma, orientan las decisiones y las acciones de los sujetos. En forma operacional, se las puede reconocer con consignas del tipo "si..., entonces..." (Catalán, 2010).

Las TS son creencias particulares, supuestos o hipótesis que crean y mantienen las personas sobre ellas y su contexto, las que, a su vez, les ayudan a comprender y comunicarse con su propio mundo. De este modo, las TS tienen una influencia sobre la forma en que el individuo comprende, planifica y posteriormente actúa en su quehacer (Flick, 2004), de allí que su estudio ayude a comprender cómo los directores y jefes técnicos entienden y definen un plan, actuando sobre él en base a sus concepciones, al tiempo que definen los factores que determinan su eficacia, delimitando el sentido y las características de este plan y su conformación.

En concordancia con lo anterior, existen distintas áreas o niveles de las TS que podrían dividirse en: a) teorías subjetivas declaradas o explícitas, las que son fácilmente 
Estudios Pedagógicos XLI, N 1: 237-256, 2015

TEORÍAS SUBJETIVAS DE DOCENTES DIRECTIVOS SOBRE FACTORES QUE DEFINEN LA EFICACIA DE LOS PLANES DE MEJORAMIENTO EDUCATIVO LEY SEP

identificables en el discurso de las personas, remitiendo a lo que éstas dicen que piensan; b) teorías subjetivas implícitas, cuyo contenido es tácito, pudiendo explicitarse por el mismo sujeto o por quien investiga (Catalán, 1997 Cit. en Castro y Cárcamo, 2012), y; c) teorías subjetivas profundas, sólo deducibles desde las "teorías subjetivas implícitas", las que, según Polanyi, formarían parte de la dimensión supuesta o virtual del conocimiento (Cit. en Castro y Cárcamo, 2012).

\subsection{PLANES DE MEJORAMIENTO, LEY SEPY SU EVALUACIÓN}

La Ley de Subvención Escolar Preferencial tiene como propósito favorecer la igualdad de oportunidades, mejorando la equidad y la calidad de la educación mediante el traspaso de recursos adicionales a los sostenedores por cada estudiante prioritario perteneciente a establecimientos adscritos voluntariamente al régimen de esta ley (MINEDUC, 2012). Para esto se debe elaborar y ejecutar un Plan de Mejoramiento Educativo (PME-SEP), en el que participa el director junto con toda la comunidad educativa con el propósito de optimizar los resultados de aprendizaje, realizando acciones en las áreas de Gestión del Currículum, Liderazgo, Convivencia y Recursos (MINEDUC, 2012).

El PME-SEP, para los establecimientos catalogados de autónomos, emergentes o en recuperación, se considera una herramienta de planificación que permite a la entidad educativa coordinar objetivos, metas y acciones para el mejoramiento de sus resultados educativos en forma continua. Éste debe abarcar desde el Primer Nivel de Transición (NT1) hasta el Octavo Básico (NB6), con especial énfasis en los alumnos prioritarios. Los alumnos prioritarios se definen como aquellos para quienes la situación socioeconómica de sus hogares obstaculiza sus posibilidades de enfrentar el proceso educativo. Por este motivo se destinan recursos adicionales por la concentración de estos estudiantes en los distintos niveles que se encuentran cursando, con el fin de "entregar más recursos donde se necesita" (MINEDUC, 2012).

En general, es posible establecer que el PME-SEP se compone de dos amplias secciones: la primera se refiere a la "Definición a Cuatro Años", etapa a largo plazo donde los establecimientos toman decisiones en torno a los sectores a trabajar para mejorar los aprendizajes SIMCE; la segunda sección lleva por nombre "Programación Anual", etapa a corto plazo donde año a año se detalla la implementación de acciones para intervenir en los sectores de aprendizaje y la gestión institucional (MINEDUC, 2008c). De esta forma, los PME-SEP constan cada año de cuatro fases que se deben ir cumplimentando: a) Diagnóstico, b) Planificación, c) Ejecución, Monitoreo y Seguimiento, y d) Evaluación (MINEDUC, 2012, 2008a).

Para cada una de estas secciones los establecimientos deben: a) definir las metas de aprendizaje para cada año de implementación del plan; b) definir las acciones de mejoramiento correspondientes a los subsectores y a la gestión de la institución educativa; c) señalar las asistencias técnicas que serán contratadas para la ejecución de las acciones comprometidas tanto en los subsectores como en materia de gestión (sólo si se contrata algún servicio), y; d) definir el sistema de monitoreo que dará seguimiento a los objetivos en dichos subsectores y en materia de gestión (Treviño, Órdenes y Treviño, 2009).

En el caso de los sectores de aprendizaje, en la Planificación Anual se deben establecer acciones específicas, las cuales se organizan en cinco ámbitos: 1) "Acciones para medir los avances en el aprendizaje"; 2) "Acciones para mejorar las estrategias de enseñanza"; 
3) "Acciones para apoyar la gestión docente en el aula"; 4) "Acciones de reforzamiento pedagógico", y; 5) "Acciones para comprometer al apoderado" (Treviño et al., 2009).

Buena parte del procedimiento de evaluación se resume en el Manual proceso de seguimiento a la ejecución de acciones del Plan de Mejoramiento Educativo y evaluación de los compromisos del sostenedor (MINEDUC, 2010a) y en el Proceso de seguimiento a la ejecución de acciones del Plan de Mejoramiento Educativo y evaluación a los compromisos del sostenedor en los establecimientos adscritos a la Ley SEP (MINEDUC, 2010b). Adicionalmente, gran parte de los aspectos observados para analizar la efectividad y la eficacia de una escuela ante un plan de mejora se encuentra en la Guía para el diagnóstico institucional de escuelas regulares con NT1 y/o NT2 del Ministerio de Educación (s/f).

Dentro de estos documentos se incluye el "proceso de monitoreo y seguimiento anual", el cual es dirigido por un supervisor técnico-pedagógico ministerial. Esta instancia conlleva la aplicación de dos pautas: a) la de seguimiento y ejecución de acciones del PME, y b) la de evaluación de requisitos, obligaciones y compromisos esenciales del sostenedor, adquiridos mediante Convenio de Igualdad de Oportunidades Excelencia Educativa (MINEDUC, 2010a, 2010b).

Este proceso, según lo establecido por el Misterio de Educación (2010a, 2010b), tiene tres objetivos: 1) seguir la ejecución de acciones del PME-SEP, mediante procesos colaborativos de análisis técnico y sobre la base de medios de verificación; 2) confirmar el cumplimiento del Convenio de Igualdad de Oportunidades, y; 3) incentivar prácticas sistemáticas de monitoreo, seguimiento y evaluación al PME-SEP (MINEDUC, 2010b). El sostenedor y la escuela deben poseer los medios de comprobación necesarios para evidenciar la ejecución de cada acción y el cumplimiento de los compromisos contraídos.

\subsection{ALGUNOS APORTES DEL MODELO DE EFICACIA ESCOLAR Y LOS FACTORES PARA SU MEDICIÓN}

Entenderemos la eficacia escolar (y el modelo de eficacia escolar) siguiendo la definición de Murillo (2003a), quien nos dice que la eficacia escolar promueve de forma duradera el desarrollo integral de todos y cada uno de sus alumnos más allá de lo que sería previsible, teniendo en cuenta su rendimiento inicial y su situación social, cultural y económica. Las investigaciones sobre las escuelas eficaces abordan dos ejes temáticos: la estimación de la magnitud del efecto escuela, y la identificación y el análisis de los factores escolares, de aula y de contexto que hacen que una escuela sea eficaz (Raczynski y Muñoz, 2005).

En un plano conceptual, y posiblemente también desde el sentido común, la efectividad puede ser comprendida como un término difuso. En este sentido, un avance importante lo realiza Cuttance en 1987 (Cit. en Arancibia, 1992) al proponer un modelo de efectividad que considera dos dimensiones principales: calidad y equidad. La primera está conformada como la media de los puntajes de cada colegio en los resultados (corregido por nivel socioeconómico), mientras que la segunda tiene relación con la capacidad de los colegios de nivelar las características de entrada de los estudiantes.

Sumado a lo anterior, el modelo de eficacia escolar ha realizado importantes aportes, entre los cuales se encuentra la definición de una serie de factores que se consideran esenciales (o interventores) para la efectividad: a) metas compartidas, consenso, trabajo 
en equipo; b) liderazgo educativo; c) orientación hacia el aprendizaje; d) clima escolar; e) altas expectativas; f) calidad del currículo; g) organización del aula; h) evaluación frecuente; i) desarrollo profesional; j) participación e implicación de la comunidad educativa, y; k) recursos e instalaciones (Bellei, Muñoz, Pérez y Raczynski, 2004).

A nivel nacional, Bellei (2010), en su estudio sobre las ATE y su vinculación con los PME, encontró dos factores comunes: a) la dimensión pedagógica-curricular, y b) la dimensión de gestión institucional, las cuales, en un análisis más profundo, muestran tener relación con el modelo de eficacia escolar. Otro aporte proveniente del modelo de eficacia escolar realizado por Murillo (2003b Cit. en Raczynski y Muñoz 2005), establece tres claves para la efectividad en la escuela: a) el principio de equidad, b) la idea de valor agregado, y c) la preocupación por el desarrollo integral del alumno. Otra forma de aproximarse a los factores característicos de la eficacia escolar es la investigación de Bellei et al. (2004) sobre escuelas efectivas en sectores de pobreza, cuyos resultados involucran aspectos de gestión institucional, compromiso y calidad profesional, vinculación con el establecimiento, enseñanza de aprendizajes y relación con apoderados.

En este punto se hace necesario aclarar que muchas veces existen problemas para diferenciar la efectividad, la eficacia y la eficiencia. Para este estudio, en general, se siguió la noción de eficacia, pues ya se contaba con los antecedentes del modelo de eficacia escolar. Ahora bien, siguiendo a Chase y Aquilano (1995), podemos definir brevemente la eficacia como la obtención de resultados deseados, y la eficacia como el logro de un resultado deseado con el mínimo de insumos (Cit. en Barrientos, 2008), mientras que la efectividad tiene relación con los efectos de una actividad y sus resultados, beneficios y consecuencias finales para una población, en virtud de los objetivos propuestos (Lam y Hernández, 2008).

$\mathrm{Al}$ analizar estos resultados es posible apreciar que ellos van en el sentido de mejorar y alcanzar resultados establecidos o propuestos. De esta manera, se cumple con una importante base para nuestra investigación, esto es, analizar los factores que los directivos identifican mediante sus teorías subjetivas, con el fin de obtener dichos resultados y lograr la eficacia o la efectividad, según como ellos muchas veces relacionan explícita e implícitamente estos conceptos. Ejemplo de lo anterior es lo mencionado por el Director de una Escuela Rural: "podría decirse que efectividad es realizar en buena forma la colocación, que será efectivo, que los aprendizajes o sea lleguen a los alumnos y los aprovechen bien".

\subsection{METAS DE EFECTIVIDAD DE LOS PLANES DE MEJORAMIENTO EDUCATIVO LEY SEP}

El Ministerio de Educación establece metas de efectividad en base a los resultados SIMCE, los cuales son proporcionales a la matrícula del establecimiento y a una diferencia significativa en relación con su puntaje SIMCE al momento de firmar el Convenio de Igualdad de Oportunidades como compromiso de mejora. Por ejemplo, en cuanto a las denominadas "metas obligatorias anuales de velocidad y comprensión lectora", incluidas en el apartado del PME-SEP de programación anual, el Ministerio de Educación sugiere un mínimo que corresponde al menos a un $90 \%$ de los estudiantes con una lectura media alta o superior (MINEDUC, 2008d). Además se considera una sección para incorporar metas optativas, las cuales pueden ser cuantitativas o cualitativas (MINEDUC, 2013; Treviño et al., 2009). 


\section{DISEÑO, METODOLOGÍA Y PROCEDIMIENTOS DE INVESTIGACIÓN}

\subsection{CONTEXTO DEL ESTUDIO}

La muestra fue conformada por 10 participantes, todos docentes directivos (directores y jefes de UTP) pertenecientes a siete de los once establecimientos educacionales polidocentes municipalizados de la comuna de Vicuña (diez de Educación Básica y uno de Educación Media). Dichos establecimientos cuentan con altos índices de vulnerabilidad y matrículas entre los 60 y 450 estudiantes al momento de la entrevista, lo que representa sobre el $50 \%$ de los estudiantes de educación municipalizada de la comuna. Además, esta muestra cuenta con cuatro de los cinco establecimientos de mayor matrícula, siendo dos de éstos del radio urbano de la ciudad, y el resto de localidades aledañas.

Dentro de los criterios de inclusión y exclusión, siguiendo el criterio de intencionalidad de la muestra, se eligen estos cargos pues son de gran incidencia en la comunidad educativa y en la elaboración de los planes de mejora educativa, siendo el jefe técnico quien se asocia a los diagnósticos de aprendizaje, y el director quien debe subir la información al portal. Además, el primero está directamente relacionado con la gestión de los aprendizajes, y el segundo con la administración y la gestión educacional.

Adicionalmente, se buscó que al menos dos docentes directivos fueran de establecimientos de sectores urbanos (tres establecimientos urbanos en total) y dos de sectores rurales (de siete en total), y que tuvieran como mínimo un año de experiencia en el cargo (entre un año y medio y treinta años). Esto con el fin de abordar aspectos administrativos y pedagógicos de la temática a investigar, junto con recabar diversas perspectivas que puedan enriquecer la información recopilada, y seguir, asimismo, un criterio de accesibilidad a la muestra.

\subsection{MÉTODO DE RECOLECCIÓN}

La técnica de recolección utilizada fue la entrevista individual a docentes, en cada una de las cuales se buscó alcanzar el criterio de saturación teórica (Flick, 2004). Las entrevistas realizadas fueron semiestructuradas. Para algunos (Valles, 2003) ésta es la más común de las entrevistas cualitativas, la cual es guiada por un conjunto de preguntas y cuestiones básicas a explorar, pero donde ni la redacción exacta, ni el orden de las preguntas está predeterminado. Con el fin de resguardar la transparencia del proceso y los derechos de los participantes, antes de la realización de las entrevistas se estableció la firma de un consentimiento informado, donde se incluyeron las características generales de la investigación y el derecho de recibir información sobre la investigación una vez terminado el proceso.

\subsection{PROCEDIMIENTO DE ANÁLISIS}

Se recurrió al análisis de contenido de las entrevistas utilizando el método de comparaciones constantes, y estableciéndose, como ya se dijo, el criterio de saturación teórica (Taylor y Bogdan, 1996). De este modo, y para profundizar en el análisis, se utilizaron las tres fases de la teoría fundamentada: codificación abierta, axial y selectiva, con el fin de codificar los contenidos, organizarlos en distintos modelos teóricos, y así llegar a un modelo final que dé 
cuenta de cómo los docentes construyen la definición de los planes de mejora y los criterios de eficacia de los mismos.

En relación con lo anterior, y con el fin de resguardar el rigor científico dentro de esta investigación, se recurrió a los criterios de credibilidad, confirmabilidad, transferibilidad o aplicabilidad. Por ello se realizó un análisis exhaustivo de los datos, se describieron los sujetos de estudio y se realizó la presentación de los resultados a los mismos. Sumado a esto, se consultó a otros investigadores sobre algunos análisis.

Para la sistematización y el tratamiento de la información recopilada durante este proceso se usó el programa computacional Atlas.ti (Muhr, 2003-2008), pues es un reconocido software para la investigación y el análisis cualitativo de los datos. Además, este programa se basa en la Grounded Theory (Strauss \& Corbin, 2002) para su fundamentación teórica, permitiendo realizar análisis congruentes con los tres procesos de codificación (Varguillas, 2007).

De esta manera, a continuación se presenta para cada apartado del análisis de resultados aquellos datos recogidos por medio de las codificaciones, mostrando principalmente los dos primeros procesos en la siguiente sección, y exponiendo en las conclusiones el proceso de codificación axial. Por último, es necesario mencionar que el proceso de análisis de las entrevistas fue realizado por el investigador, quien para la transcripción de algunas entrevistas contó con el apoyo de sujetos externos bajo su cargo, y para la codificación de todas las entrevistas consultó a otros profesionales con el propósito de validar el proceso.

\section{ANÁLISIS DE RESULTADOS}

\subsection{DEFINICIÓN DE PLAN}

En los siguientes párrafos se da cuenta de uno de los objetivos específicos de esta investigación, a saber, describir las definiciones del plan de mejoramiento educativo. En este sentido, todos los docentes ven el PME-SEP como un conjunto de objetivos o estrategias que se estructuran dentro del establecimiento para alcanzar las metas propuestas y/o los resultados previstos de manera programada, vinculándose principalmente con la mejora de los aprendizajes, tal como lo menciona la siguiente entrevistada:

"Un Plan de Mejoramiento Educativo es un instrumento técnico que permite desarrollar una programación anual, que considera acciones y metas planificadas, a fin de lograr mejores resultados de aprendizajes de los estudiantes y mejores resultados de la gestión escolar, ya que está diseñado para que la escuela misma aprenda cómo enfrentar las exigencias de mejoramiento..." (Directora, Escuela Rural).

Ahora bien, la definición del plan no está orientada sólo al mejoramiento de los aprendizajes, sino también a la gestión interna y administrativa, tal como lo enuncia este docente directivo:

“Es un proceso de mejoramiento de los aprendizajes y de..., tanto administrativo, como técnico de un colegio...” (Jefe Técnico, Escuela Rural). 
Esta estrategia orientada a la mejora es consensuada, partiendo de un diagnóstico y ajustándose al contexto interno y externo (tanto escolar, como social) en sus acciones, buscando desarrollar las actividades de manera real y en forma participativa, convirtiéndose en una herramienta fundamental en la actualidad:

“...un plan de mejoramiento educativo para una escuela es fundamental porque, en primer lugar, se elabora dentro de la escuela con la participación de todos los miembros activos de la comunidad de la escuela...” (Director, Escuela Rural).

En base a los procesos de codificación abierta y selectiva, se obtuvo el siguiente diagrama que resume los factores relacionados con la definición de un PME-SEP. Éste se ha elaborado a partir de un código general de definición del plan junto con tres categorías generales.

Figura $N^{\circ}$ 1. Codificación axial, definición del plan

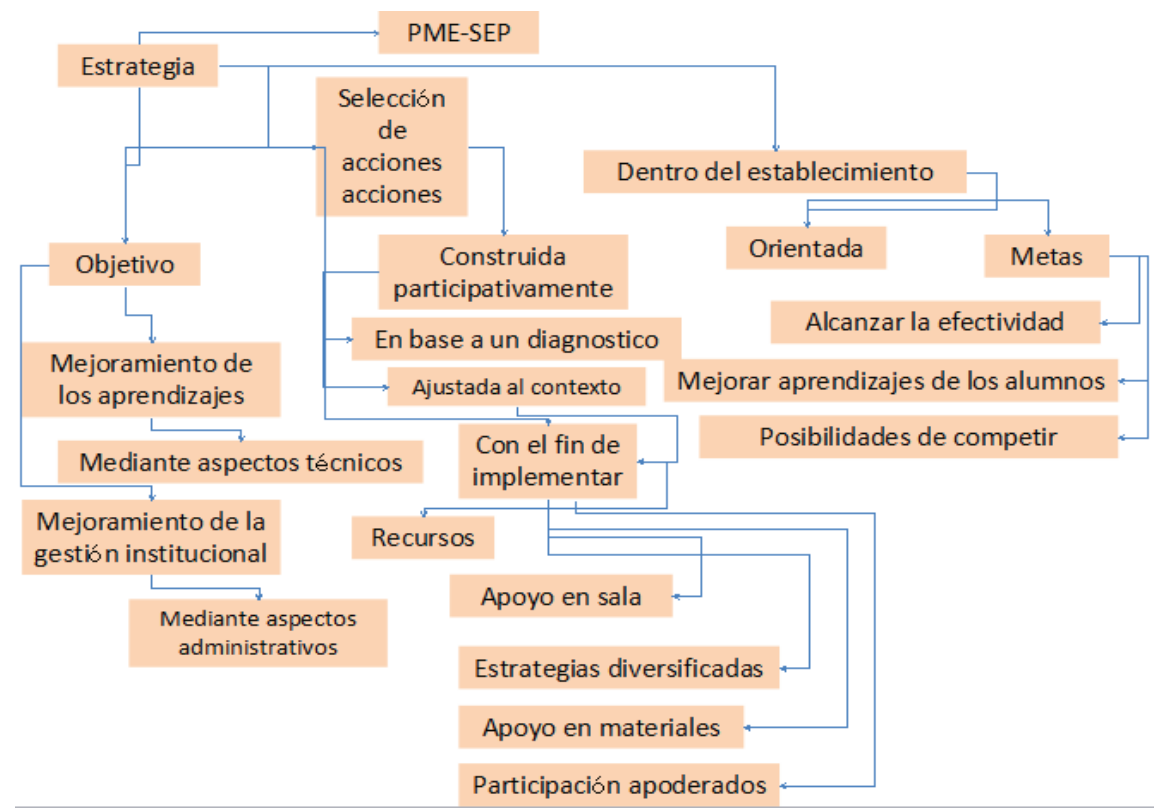

Fuente: elaboración propia.

A grandes rasgos, el PME-SEP es considerado como una estrategia que tiene tres características generales: a) tener objetivos claros, divididos en el mejoramiento de los aprendizajes y el mejoramiento de la gestión institucional, b) estar constituida por acciones, las cuales se construyen participativamente, en base a un diagnóstico y con el fin de conseguir recursos, y; c) estar dentro del establecimiento, orientándose a metas con resultados establecidos. 


\subsection{CONCEPCIONES BAJO LA EFICACIA DE LOS PLANES}

"La escuela para que sea efectiva tiene que estar organizada, y bien organizada con todos sus medios de planificación, todos sus indicadores que le van dando a uno el camino de lograr el éxito" (Jefe Técnico, Escuela Rural).

$\mathrm{Al}$ analizar los relatos de los docentes es clara la importancia de la organización para alcanzar la efectividad y la eficacia. Esta capacidad de contar con una escuela organizada se basa en factores tanto administrativos (tiempo-espacios, recursos tecnológicos, etc.), como profesionales (disposición del profesor, trabajo en equipo, etc.) y curriculares (planificaciones al día, liderazgo pedagógico, foco en los aprendizajes, etc.). Éstos, al interactuar entre sí, fortalecen la capacidad de la escuela para responder a las metas y los objetivos de los PME-SEP, ya sea internos o externos.

También es posible ubicar la identificación con el establecimiento como otro gran factor que va de la mano con la organización. Esta identificación pareciera ser necesaria en todos los actores del proceso para alcanzar la efectividad, y se asocia fuertemente con el compromiso que el individuo desarrollará con respecto a la meta del establecimiento y a la meta no expuesta en los planes de conseguir seres humanos integrales.

"Que vibren, que tengan la identidad de la escuela, sino que toda la escuela (sic), tanto alumnos como apoderados, profesores, realmente sientan identidad, que vivan el colegio" (Director, Escuela Rural).

"Una gama de proyectos que hacen que también el niño se desarrolle integralmente, no solamente la parte cognitiva” (Directora, Escuela Urbana).

Ahora bien, la organización del plan tiende a fundirse en sus áreas, sobre todo si se analiza desde la perspectiva de cumplir las metas centradas en el aprendizaje de calidad según lo impuesto por el Ministerio de Educación. De esta forma, se considera que la planificación de la clase es central desde un aspecto curricular, sin embargo, ésta es insuficiente si no se cuenta con los recursos materiales y tecnológicos necesarios. A su vez, todo esto tiende a la desarticulación si no se cuenta con el trabajo en equipo, un buen clima escolar y el liderazgo directivo, todos aspectos que se encuentran asociados a las áreas de convivencia y liderazgo.

En cuanto a la organización de los recursos, los directores y jefes técnicos han decidido en sus unidades educativas dividir los recursos en dos grandes áreas, siguiendo la estructura de la plataforma PME-SEP: 1) recursos humanos y 2) recursos materiales. Este último es el que presenta una tendencia más clara, pues, por un lado, se ha dado un fuerte impulso a la compra de tecnología con la implementación de las TICs en diversos espacios y la implementación de material didáctico acorde a las nuevas tecnologías, y por otro lado, se ha considerado la mantención de materiales de oficina dentro del establecimiento, tales como tintas y resmas suficientes, lápices, plumones, carpetas, etc., elementos que se consideran necesarios para el desarrollo de las clases y el trabajo docente, bajo el precepto clave de que todos los estudiantes cuenten con las herramientas requeridas para aprender.

Refiriéndonos a los recursos humanos, llama la atención la presencia de dos tendencias dispares pero claras: la ampliación horaria de docentes que pertenecen a la unidad educativa, bajo la idea de contar con el personal idóneo para el trabajo, pero enfrentándose 
a la necesidad de que éstos dispongan, a su vez, de mayor tiempo, o bien, aspirar a la contratación de personal nuevo que supla las carencias que el establecimiento posee, las cuales tienen relación con aspectos que muchas veces se encuentran fuera de la formación docente o rebasan la mera ampliación de contratos (psicólogos, asistentes sociales, psicopedagogos, profesores para reforzamiento en aula).

Al hablar de mejora y efectividad, en casi todos los casos, las primeras concepciones que toman forma tienen relación con el mejoramiento de los aprendizajes y/o la calidad de los mismos. Éste es el principal objetivo de la Ley 20.248 (MINEDUC, 2008a, 2008c), la cual define, a su vez, la efectividad como el cumplimiento de esta meta, tal como lo muestran las siguientes citas:

"Podría decirse que efectividad es realizar en buena forma la colocación, que será efectivo, que los aprendizajes o sea lleguen a los alumnos y los aprovechen bien” (Director, Escuela Rural).

"Un PME es efectivo cuando se ha logrado cumplir con las metas de efectividad que se han comprometido [tanto] a nivel de Comunidad educativa como con el MINEDUC" (Directora, Escuela Rural).

Dentro de la gestión curricular destaca la idea de que todos los estudiantes tienen la misma posibilidad de aprender, de manera tal que se albergan altas expectativas en relación con sus capacidades y oportunidades, lo que es un aspecto que se vincula al liderazgo. De esta manera, se articula la concepción de que la gestión curricular está basada en: alcanzar la mejora de los aprendizajes con objetivos a corto y largo plazo (objetivo plan y efectividad), lograr una planificación oportuna de las clases, la que debe ser diaria y vincularse con la óptima utilización del tiempo en el aula y con el hecho de que el profesor sepa lo que tiene que hacer. Otro aspecto importante de la gestión curricular es la realización del reforzamiento pedagógico, el cual debe ser específico a la materia y a la cantidad de alumnos que lo necesitan, con el objeto de nivelar a aquellos más descendidos, trabajando en conjunto el profesor de aula con el profesor de reforzamiento, poniendo el foco en las principales asignaturas, y contando con el apoyo y el monitoreo de la unidad técnica.

Igualmente, tanto en lo que respecta a la gestión curricular como al liderazgo, y desde allí a todas las áreas del establecimiento, se ha constado la importancia del monitoreo y la evaluación constante. En esta dirección, el monitoreo debe abarcar tanto a las acciones realizadas como a los alumnos y sus aprendizajes en forma individual, de manera tal que dicho aprendizaje resulte cuantificable, con el fin de poder analizar los avances y también establecer medidas al respecto. Estas instancias de monitoreo han sido vistas como instancias de autoevaluación, lo que implica reuniones, revisión de estados de avance, y análisis de resultados de pruebas y/o instrumentos aplicados. A su vez, tales instancias han sido vistas como mediciones externas, como lo son los resultados SIMCE o los informes del Plan de Apoyo Compartido (PAC), los cuales están orientados al aprendizaje de los alumnos, o a la visita de asesores técnico-pedagógicos que han realizado evaluaciones del estado de avance del PME-SEP, o visitas de la triada PAC que se realizan mensualmente.

Otra visión del plan que es compartida por los establecimientos, y que se asocia directamente con la efectividad de éstos, es la capacidad de unir el PME-SEP -vincularlo- a otras estrategias o actividades institucionales. En este sentido, buscan fortalecer el cumplimiento del plan sobre la base de las capacidades que ya posee y, al mismo tiempo, potenciar sus 
Estudios Pedagógicos XLI, N 1: 237-256, 2015

TEORÍAS SUBJETIVAS DE DOCENTES DIRECTIVOS SOBRE FACTORES QUE DEFINEN LA EFICACIA DE LOS PLANES DE MEJORAMIENTO EDUCATIVO LEY SEP

deficiencias, optimizando tiempo y recursos. En esta lógica es importante el planteamiento de las escuelas con respecto al PAC, quienes fusionan ambas iniciativas sobre todo en los aspectos curriculares, principalmente en las planificaciones y el acompañamiento al aula.

"Junto con los supervisores, con el ATP (Asesor Técnico Pedagógico) que se llama ahora, enlazamos la SEP con el PAC, porque tanto como el PAC tiene materiales, pero no todos, así que enlazamos acciones, del PAC pasamos acciones al plan de mejora y de ahí ya conjugamos los dos juntos y ha resultado efectivo" (Director, Escuela Rural).

En base a los análisis realizados en los procesos de codificación abierta y axial, donde se trabajó con un total de 27 categorías generales, se obtuvo el siguiente modelo que muestra los principales factores asociados con la eficacia dentro de los PME-SEP. En la Figura $\mathrm{N}^{\circ} 2$ se establecen cuatro niveles de factores en cinco círculos concéntricos, desde los más generales (círculo más lejano al factor central) a los más específicos (círculo más cercano al concepto central), los cuales rodean al factor central de "eficacia del plan de mejoramiento educativo (eficacia PME-SEP)". Estos factores son rodeados, a su vez, por seis metas, ejes y/u objetivos que se plantea conseguir y desarrollar en el plan de mejoramiento, de modo tal que el círculo central engloba cinco tipos de factores primordiales para el PME-SEP, los que son: 1) factores orientados al currículum; 2) factores orientados a la estructuración, la organización y el liderazgo; 3 ) factores orientados a los recursos, los cuales se consideran la base para alcanzar el éxito de la mayoría de los demás factores; 4) factores orientados a las características profesionales y personales, y; 5) factores humanos y de convivencia, los últimos más relacionados con características de los alumnos y los apoderados.

Como ya se mencionó, para que sea posible la existencia de esta serie de factores, se considera un conjunto de objetivos del plan, los que funcionan tanto a modo de metas a alcanzar con la ejecución, como de bases para su formulación. Desde un comienzo se supone la necesidad de proponer objetivos claros y factibles de realizar, siendo una de las características definitorias de dicha ejecución el hecho de plantear los planes con el propósito de afectar positivamente a todos los alumnos, principalmente centrando esta acción en mejorar la calidad de los aprendizajes y de la enseñanza misma, con lo que se lograría el cumplimiento de las metas del SIMCE. Claro está que todos estos objetivos buscan generar la capacidad de responder a demandas internas y externas, lo que en sí posibilitaría la creación de acciones para el PME-SEP.

$\mathrm{Al}$ referirnos al ámbito del currículum, se observa una fuerte integración de conceptos, considerando esta área como el centro del plan, ya que desde la compra de insumos al liderazgo se orienta hacia la mejora de aprendizajes y, consecuentemente, al aumento de puntajes SIMCE. Desde esta perspectiva toma vital importancia el buen uso del tiempo dentro del aula, lo que significa una estructuración clara de la clase, para lo cual se hace necesario planificar diariamente el trabajo. Asociado con lo anterior, el monitoreo de los aprendizajes adquiere un gran papel en la eficacia del plan, pues se espera de éste que sea acorde a las clases y que busque la reflexión del estudiante. Otra forma de monitoreo es el acompañamiento al aula, esta estrategia se considera clave para la evaluación y la retroalimentación del desempeño del docente, además de una forma de crecimiento profesional, ya sea entre pares o desde el equipo directivo hacia los docentes.

Desde la gestión curricular también se establece la existencia de mecanismos de reforzamiento educativo para los alumnos que lo necesiten, fundamentalmente en cursos 
Figura $N^{\circ}$ 2. Codificación axial eficacia PME-SEP

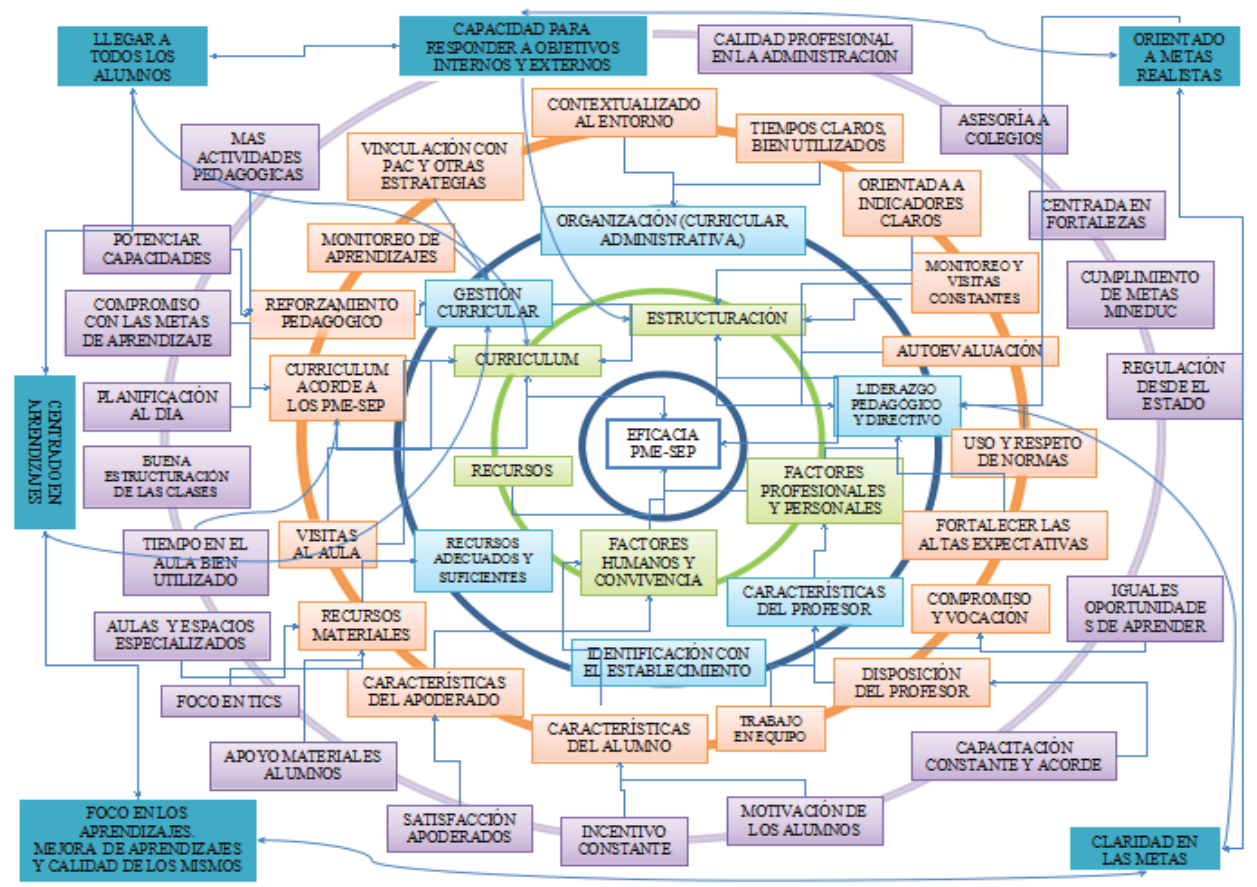

Fuente: elaboración propia.

considerados de importancia crítica por los establecimientos. Estas instancias ocurren dentro y fuera del aula, ya sea como repaso de asignaturas o de talleres que aumentan la oferta educativa, y tienen como meta acrecentar las posibilidades de aprender de los estudiantes, confiando en que todos tienen las mismas oportunidades de aprender, y/o potenciando sus capacidades por igual.

En un lugar intermedio entre los aspectos curriculares y de estructuración se encuentra la idea de vincular el PME-SEP a otras estrategias de aprendizaje y/o de gestión, como lo son el Plan de Apoyo Compartido (PAC) y el Proyecto Educativo Institucional (PEI), entre otras. La concepción subyacente en este punto es la de proveer recursos y reducir los esfuerzos para cumplir las metas, focalizando todas las voluntades de mejora en una estrategia común que agrupe otras estrategias afines. De esta forma, también se han vinculado otras iniciativas que se desarrollan dentro de los establecimientos, como lo son el plan lector y el cálculo mental (como estrategias de mejora específica), o acciones como la certificación medioambiental o el nexo con otras instituciones que intervienen en los establecimientos, tales como el Servicio Nacional para la Prevención y Rehabilitación del Consumo de Drogas y Alcohol (SENDA).

Desde el marco de la estructuración se instala una serie de acciones y factores que influyen en todas las áreas del establecimiento, con el objetivo de mejorar el aprendizaje al tiempo que se genera la capacidad de responder a los problemas. Es así como la organización, principalmente curricular y administrativa, se centra en la buena delimitación y el uso del 
Estudios Pedagógicos XLI, N 1: 237-256, 2015

TEORÍAS SUBJETIVAS DE DOCENTES DIRECTIVOS SOBRE FACTORES QUE DEFINEN LA EFICACIA DE LOS PLANES DE MEJORAMIENTO EDUCATIVO LEY SEP

tiempo, así como en la creación de un plan contextualizado al entorno, considerando como base las fortalezas del establecimiento y su contexto.

Además de lo anterior, la organización del plan debe estar orientada a indicadores, metas claras y alcanzables, que se establecen a partir de las exigencias ministeriales, y que a su vez son monitoreadas por el MINEDUC, ya sea por los resultados SIMCE o ATP. De lo anterior se desprende la necesidad de contar con asesoría, visita y monitoreo constante a los establecimientos, la cual debe ser satisfecha por profesionales competentes con conocimientos acerca de la administración de recursos SEP y de la elaboración de planes de mejoramiento, función que debe ser complementada por los ATP.

Un último aspecto a mencionar respecto de la estructuración es la fuerte importancia otorgada al liderazgo directivo y pedagógico. En este escenario, el director y el equipo técnico deben ser líderes claros que ayuden a mantener altas expectativas a nivel del trabajo en el aula, y adicionalmente este equipo es el responsable de mantener y dirigir el monitoreo y la autoevaluación constante del PME-SEP, junto con velar por los aprendizajes de los estudiantes.

Los recursos son factores que se consideran la base para la eficacia del plan, esto es, su alta ejecución, por lo cual tienen que ser adecuados y suficientes en calidad y cantidad, dividiéndose en materiales y humanos. Los primeros contemplan la implementación de espacios educativos para alcanzar mejores aprendizajes, una fuerte dotación que incluye el uso de las TICs y el apoyo con materiales para el desarrollo de las actividades en aula. Los segundos están orientados a la creación de equipos multidisciplinarios (profesores de reforzamiento, psicólogos, psicopedagogos, asistentes sociales) que complementen y enriquezcan la oferta que el colegio tiene para ofrecer a su alumnado, y que intenten apoyar tanto los aprendizajes como el desarrollo afectivo y social de los estudiantes.

Los factores profesionales y personales están orientados, principalmente, a las características del profesor, del cual se espera vocación y compromiso con los estudiantes y con su profesión. Esto se asocia a una buena disposición para el trabajo dentro del establecimiento y el desempeño en equipo, junto a las posibilidades de capacitación que puedan resultar del mismo.

La identificación con el establecimiento es un factor que es compartido tanto por los docentes como por los apoderados y los alumnos. Esta característica se relaciona con los factores humanos y de convivencia que están vinculados a rasgos como la motivación del alumno hacia el aprendizaje, la autosuperación y la necesidad de incentivo permanente, ya sea material, emocional o social. A su vez, la identificación se enlaza con las características de los apoderados, lo que generalmente se desprende de su apoyo a la labor del establecimiento, de su sentido de pertenencia hacia éste, así como de la satisfacción que éstos tengan respecto del trabajo realizado en el centro educativo.

En suma, un modelo para entender la eficacia de los PME-SEP contempla la conjunción de cinco tipos de factores, siendo primordiales el diseño del currículum con foco en los aprendizajes y una sólida estructuración, con claros liderazgos directivos y pedagógicos. A éstos se suman los factores humanos y de convivencia, y los factores profesionales y personales que se sustentan en la motivación hacia el aprendizaje y en la identificación con el establecimiento. En especial, este último factor se articula a la ejecución del plan, tanto en su carácter material como humano, pues sin él no se sustentarían las otras cuatro dimensiones. A su vez, el plan de mejoramiento se define a partir de las metas propuestas, con énfasis en aspectos curriculares, en pos de la mejora de los aprendizajes y de la adquisición de recursos. 
Figura $N^{\circ}$ 3. Codificación selectiva eficacia PME-SEP

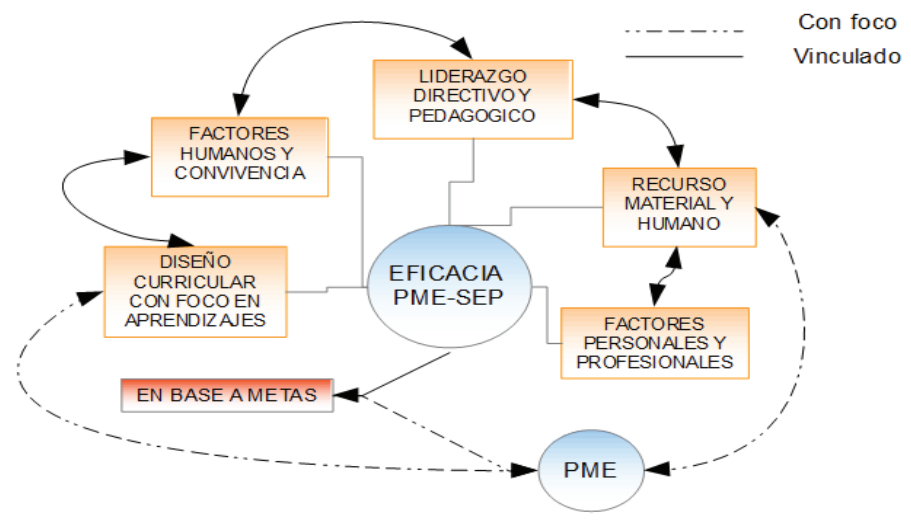

Fuente: elaboración propia.

\subsection{ANÁLISIS DE RESULTADOS SIMCE EN CONTRASTE CON LAS TEORÍAS SUBJETIVAS SOBRE LA EFECTIVIDAD DE LOS PLANES DE MEJORA}

Otro objetivo general de la presente investigación fue analizar si las posibles diferencias en las teorías relacionadas con la efectividad de los PME-SEP se vinculaban con los resultados SIMCE. Este análisis no tiene ningún fin cuantitativo, sólo tiene por objetivo observar alguna relación entre las teorías subjetivas de los directivos sobre la eficacia de los planes de mejoramiento educativo, en contraste con el desempeño SIMCE obtenido en estos últimos años en sus establecimientos, pues, como se vio anteriormente, mediante estas teorías subjetivas ellos buscan alcanzar mejoras traducidas de alguna manera en resultados. En la siguiente tabla se muestran los resultados obtenidos por los establecimientos donde trabajan los directivos entrevistados.

Tabla $N^{\circ}$ 1. Resultados SIMCE de las escuelas de los directivos entrevistados

\begin{tabular}{|c|c|c|c|c|c|c|c|c|c|c|}
\hline & \multicolumn{3}{|c|}{$4^{\circ}$ BASICO 2012} & \multicolumn{3}{|c|}{$4^{\circ}$ BASICO 2011} & \multicolumn{3}{|c|}{$4^{\circ}$ BASICO 2010} \\
\hline & & $\begin{array}{l}\text { Comprensión } \\
\text { de Lectura }\end{array}$ & Matemática & $\begin{array}{c}\text { Historia, } \\
\text { Geografia y } \\
\text { Ciencias } \\
\text { Sociales }\end{array}$ & $\begin{array}{c}\text { Comprensión } \\
\text { de Lectura }\end{array}$ & Matemática & $\begin{array}{c}\text { Historia, } \\
\text { Geografía y } \\
\text { Ciencias } \\
\text { Sociales }\end{array}$ & $\begin{array}{c}\text { Comprensión } \\
\text { de Lectura }\end{array}$ & Matemática & $\begin{array}{c}\text { Historia, } \\
\text { Geografia y } \\
\text { Ciencias } \\
\text { Sociales }\end{array}$ \\
\hline RDE & Urbano & 285 & 296 & 280 & 290 & 317 & 291 & 282 & 270 & 269 \\
\hline JTM & Urbano & 284 & 292 & 267 & 255 & 235 & 252 & 263 & 260 & 252 \\
\hline EVC & Urbano & 249 & 244 & 239 & 272 & 280 & 225 & 242 & 228 & 225 \\
\hline$J V L$ & Urbano & 251 & 243 & 234 & 246 & 235 & 236 & 246 & 226 & 236 \\
\hline JAN & Urbano & 205 & 190 & 207 & 267 & 239 & 220 & 238 & 235 & 220 \\
\hline LGA & Urbano & 238 & 239 & 218 & 239 & 222 & 223 & 249 & 222 & 223 \\
\hline ABM & Rural & 254 & 253 & 229 & 0 & 0 & 0 & 0 & 0 & 0 \\
\hline
\end{tabular}

Fuente: MINEDUC, 2012-2013.

Si tomamos como referencia el año 2010 y lo comparamos con el 2012, es posible dividir dos grandes grupos de escuelas: en primer lugar, están las escuelas que han 
tenido resultados positivos y/o al alza, sin importar alguna fluctuación de por medio (se encuentran en las primeras tres filas de la tabla); en segundo lugar, están las escuelas que han tenido resultados a la baja, aunque pudieran presentar un alza de puntaje en algún momento (lugares 4, 5 y 6 de la tabla), y; en tercer lugar, tenemos un establecimiento que sólo presenta resultados en la última aplicación, pues en los años anteriores muy pocos alumnos rindieron esta prueba, por lo cual no fue contemplado mayormente en los análisis.

En forma general, se presentaron gran número de visiones similares sobre la efectividad en los PME-SEP. De esta manera, todos concuerdan en la importancia del reforzamiento educativo, de los recursos materiales y humanos, de la disposición del profesor, de la buena convivencia escolar y del trabajo coordinado. No obstante, sí se evidencian algunas diferencias, por lo que haremos hincapié en ellas.

"Es un factor importante el ver cómo el profesor le da la importancia debida a lo que el colegio quiere instalar, porque en el fondo tú propones una acción pero quien la va a ejecutar son los profesores, y si hablamos del profesor, hablamos del liderazgo nuevamente en la sala, hablamos del tipo de niños, hablamos del compromiso que el profesor tenga frente a la acción" (Jefe Técnico, Escuela Urbana).

Se revela el modo en cómo los docentes directivos teorizan acerca de la visión del profesor dentro del plan. En este sentido, dos de los establecimientos con mayores puntajes designan tareas o labores específicas sobre algunas acciones al docente de aula, entregándole la responsabilidad completa sobre la ejecución de las mismas; mientras que dos de los establecimientos con menores resultados tienden a centralizar la responsabilidad de la ejecución sobre el equipo directivo, pues reconocen la gran cantidad de tareas que ya tiene el docente en la sala de clases.

"Profesores que han asumido la responsabilidad como corresponde ya, no necesitamos nosotros agentes externos para que..., venir a mejorar nuestras acciones, sino que con la calidad de los profesores" (Jefe Técnico, Escuela Rural).

"En primer lugar el compromiso de los docentes con la escuela, el compromiso de los apoderados, el compromiso de los alumnos, el apoyo que deben tener todos los profesores por parte del director y el UTP, es decir, entregarles autonomía” (Director, Escuela Rural).

Sumado a lo anterior, en los establecimientos con mejores resultados se ha observado una fuerte tendencia a la organización y al liderazgo directivo como base para la formulación del PME-SEP. Este liderazgo es visto como la capacidad de delegar y guiar a la institución, manteniendo el foco en mejorar los aprendizajes, lo que se traduce en aumentar los resultados SIMCE.

"Esto habla de la calidad del profesional que está acá, en esta área directiva, entonces si nosotros vemos que ése es el nicho debiéramos de alguna forma fortalecerlo, fortalecerlo de manera de ir entregando más responsabilidades" (Director, Escuela Urbana).

A su vez, los establecimientos que han logrado menores resultados centran sus perspectivas en la capacidad de vincular a los profesores con sus alumnos. Adicionalmente, 
sitúan como factores primordiales la identificación con el establecimiento y el mejoramiento del compromiso del docente.

Una teoría manejada por los tres establecimientos que han presentado menores desempeños es la atribución de los resultados, especialmente los más bajos, a las características sociales y/o biológicas de los alumnos, tales como problemas físicos, intelectuales, de drogadicción, conflictos asociados al sector poblacional de alto riesgo, entre otros, y además se cree que son escasas las posibilidades de modificarlos o contrarrestarlos. Por el contrario, los establecimientos con mejores puntajes tienden a reconocer la existencia de problemáticas en sus estudiantes, pero creen que, a pesar de ellas, "todos tienen las mismas posibilidades de aprender". Por último, es importante mencionar que estos resultados guardan claras similitudes con otros estudios que observaron, en escuelas de alta vulnerabilidad, diferencias en el discurso sobre resultados de aprendizaje (Volante y Müller, 2006).

\section{CONCLUSIÓN Y DISCUSIÓN}

Pareciera ser que más allá de las posibles diferencias que puedan tener los directores y jefes técnicos sobre cómo ven el plan de mejoramiento educativo y los factores que definen su alcance y eficacia, existen ciertos puntos de común acuerdo que son valorados como ejes principales del PME-SEP, tanto en forma explícita como implícita.

Uno de los principales factores es la identificación con el establecimiento. En esta dirección, tanto los establecimientos que han obtenido aumentos considerables en sus puntajes SIMCE como los que no, han atribuido un valor destacado a la vinculación que se logra de parte del alumno, del profesor y del apoderado. En este sentido, se le asigna significancia a la identificación tanto en relación con el aumento de los aprendizajes, como en relación con la motivación para aprender o participar. A su vez, la identificación la relacionan con la posibilidad de dejar alguna marca o apropiación particular en los estudiantes.

En relación con lo anterior, parece ser el sentido o meta final del plan el hecho de cumplir con lo exigido por el Ministerio de Educación, dicho de otra forma, se espera cumplir con mejorar la calidad de los aprendizajes, lo que dentro de los establecimientos implica aumentar los puntajes SIMCE. Esto, en muchas ocasiones, parece generar tensión respecto de la importancia y el enfoque que se le da a la identificación con el establecimiento, lo que para los actores está más vinculado con un desarrollo valórico y/o personal, que con un desarrollo académico. Para que esto sea posible se considera de vital relevancia el trabajo en el aula, con un fuerte foco en el fortalecimiento de la planificación y la correcta utilización de los tiempos dentro de la sala de clases, sumado a la existencia de materiales y profesionales acordes a las necesidades con que cuenta el establecimiento.

De este modo, el modelo resultante de los procesos de codificación, donde cinco factores resultan clave para explicar la eficacia de los planes de mejoramiento, nace desde una concepción donde se define el PME-SEP como una estrategia orientada a alcanzar las metas propuestas, mediante la obtención de recursos materiales y humanos, con énfasis en los aprendizajes y el diseño curricular. A esto se añade la importancia de la estructuración de un liderazgo directivo y pedagógico sólido, la existencia de factores profesionales, humanos y de convivencia, elementos que son esenciales para generar compromiso e identificación con el establecimiento y el plan de mejoramiento. 
Al comparar la formulación de este modelo con los resultados SIMCE obtenidos por los distintos establecimientos estudiados, no se observan mayores diferencias en la estructura del plan o en la importancia que los docentes directivos le dan a uno u otro factor. Sin embargo, existen matices que parecen sustentar justificaciones respecto de los resultados en los aprendizajes de los estudiantes. Éstos se relacionan con la motivación del educando, el apoyo familiar dentro del proceso educativo, las "dificultades biológicas o sociales" del alumnado, la identificación con el establecimiento, la vocación del profesor y el recurso material y humano necesario. Estos factores son vistos como favorables o positivos en las escuelas con mayores resultados, mientras que se observan como negativos o desfavorables en las escuelas con menores resultados.

Finalmente, al momento de analizar la estructuración resultante de los factores asociados a la eficacia del plan, llama la atención cómo los directores y jefes técnicos concordaron en sus teorías con los planteamientos del modelo SACGE (Sistema de Aseguramiento de la Calidad en Gestión Escolar) que, desde el 2005, propone cinco áreas cuyo centro se ubica en la gestión curricular, la que a su vez se encuentra vinculada con el liderazgo, la convivencia escolar y el apoyo dado a los estudiantes, los recursos y los resultados (González, 2012). Este modelo pareciera ser una de las bases del Ministerio de Educación para la elaboración de los actuales PME-SEP pues, como se menciona en las Orientaciones técnicas para la elaboración del Plan de Mejoramiento Educativo (MINEDUC, 2013), las sugerencias para formular dicho plan se vinculan al SACGE, junto a otros estudios orientados al análisis de las "buenas prácticas".

En base a lo mencionado es posible desprender una serie de conjeturas. Resulta de importancia considerar que parte de la estructura del SACGE es congruente con la visión de los docentes directivos sobre la eficacia de los planes de mejoramiento, o que al menos sus áreas resultan concordantes con esa visión, o bien, que este modelo influyó en la actual concepción que los docentes directivos tienen de un plan orientado a la eficacia, ya sea por su propia perspectiva o por la dependencia institucional de las actuales orientaciones para la estructuración del plan. Estructura con la cual los entrevistados parecieran estar conformes, ya que no surge ni en los contenidos ni en el análisis del proceso evidencia que indique rechazo o resistencia a los términos de estas orientaciones.

Lo anteriormente dicho nos lleva a pensar en la profundidad de estas concepciones, pues las teorías subjetivas recogidas durante la investigación pueden ser parte de las iniciativas introducidas desde el marco regulatorio (MINEDUC) con anterioridad a la experiencia de mejora, las cuales, con el paso de los años y los distintos procesos de internalización experimentados por los directivos, pasaron a formar parte de sus propias concepciones explícitas e implícitas sobre gestión escolar. Sin embargo, estas teorías siguen siendo interesantes a la hora de estudiar cómo se estructuran e interpretan las iniciativas de mejora para alcanzar la eficacia y/o efectividad escolar, en contextos y situaciones específicas, considerando las TS como orientaciones hacia la acción sobre la elaboración e implementación del PME-SEP.

En términos de aportes y límites de esta investigación, se ha obtenido información de primera fuente sobre cómo los establecimientos de la comuna de Vicuña construyen sus concepciones respecto al Plan de Mejoramiento Educativo y los criterios que definen la eficacia del mismo, generando bases para establecer un futuro modelo de eficacia que sea factible de aplicar desde la propia perspectiva de los participantes de la intervención, además de corroborar cómo los docentes directivos atribuyen o no importancia a factores 
como el SIMCE, los resultados, los aprendizajes, la experiencia, la matrícula y el uso de las tecnologías, ente otros. En futuras investigaciones se espera comparar estos resultados con investigaciones de otro tipo, y crear instrumentos o indicadores para analizar estos factores en distintos establecimientos.

\section{REFERENCIAS BIBLIOGRÁFICAS}

Arancibia, V. (1992). Efectividad escolar: Un análisis comparado. Estudios Públicos, (47), 101-125. Recuperado el 17 de diciembre de 2012 desde www.cepchile.cl/dms/archivo_1819_1298/ rev47_arancibia.pdf

Barrientos, E. (2008). Didáctica de la educación superior I. Lima: Universidad Nacional Mayor de San Marcos. Recuperado el 18 de julio de 2013 desde https://mail-attachment.googleusercontent. $\mathrm{com} / \mathrm{attachment} / \mathrm{u} / 0 / \mathrm{ui}=2 \& \mathrm{ik}=62 \mathrm{c} 13 \mathrm{a} 74 \mathrm{~b} 3 \&$ view $=$ att $\&$ th $=140 \mathrm{dcb} 6 \mathrm{~cd} 4 \mathrm{f} 6 \mathrm{a} 551 \&$ attid $=0.1 \& \mathrm{disp}=\mathrm{saf}$ e\&realattid=f_hl34suk80\&zw\&saduie=AG9B_P8wq-67Eha5u6SUjGjvMppb\&sadet=13780928422 60\&sads $=\mathrm{jAsF} 4 \mathrm{~J} 0 \mathrm{~m} 2 \mathrm{TGQd}$ dnxPEBwh7ong

Beilli, C. (2010). Asistencia Técnica Educativa en Chile: ¿Aporte al mejoramiento escolar? Santiago: Ocho Libros.

Bellei, C., Muñoz, G., Pérez, L. y Raczynski, D. (2004). ¿Quién dijo que no se puede? Escuelas efectivas en sectores de pobreza. Santiago: UNICEF-MINEDUC.

Castro, P. y Cárcamo, R. (2012). Cambio de teorías subjetivas de profesores respecto a la educación en valores. Subjetividad y Procesos Cognitivos, vol.1 (1), 17-42. Recuperado el 19 de julio de 2013 desde http://www.scielo.org.ar/pdf/spc/v16n1/v16n1a01.pdf

Catalán, J. (2010). Teorías subjetivas: Aspectos teóricos y prácticos. La Serena: Editorial Universidad de La Serena.

(2004). Formación inicial de educadoras de párvulos: Un estudio de caso desde las teorías subjetivas de formadores y formadoras. Revista Iberoamericana de Educación, vol.33 (5), 1-15. Recuperado el 17 de diciembre de 2012 desde http://www.rieoei.org/deloslectores/738Catalan. PDF

Flick, U. (2004). Introducción a la investigación cualitativa. Madrid: Ediciones Morata.

González, J. (2012). Los planes de mejoramiento educativo de la Ley SEP. Su función en el proceso de enseñanza-aprendizaje y en el desarrollo local. Recuperado el 13 de junio de 2013 desde http://www.munitel.cl/eventos/seminarios/html/DOCUMENTOS/2012/XXXVIII_ESCUELA_DE_ CAPACITACION_CHILE/COQUIMBO/PPT21.pdf

Lam, R. y Hernández, P. (2008). Los términos: eficiencia, eficacia y efectividad ¿son sinónimos en el área de la salud? Revista Cubana Hematol Inmunol Hemoter, vol.24 (2), 1-6. Recuperado el 15 de junio de 2012 desde http://scielo.sld.cu/scielo.php?script=sci_arttext\&pid=S086402892008000200009\&lng=es\&nrm=iso

MINEDUC (2013). Orientaciones técnicas para la elaboración del Plan de Mejoramiento Educativo. Santiago: Ministerio de Educación. Recuperado el 30 de junio de 2013 desde http:// www.comunidadescolar.cl/documentacion/carta_ministro/4.\%20Plan $\% 20 \mathrm{de} \% 20 \mathrm{Mejoramiento} \% 20$ Educativo\%20del\%20Establecimiento/1.3.-\%20Registro\%20Plataform

MINEDUC (2012). Subvención Escolar Preferencial. Santiago: Ministerio de Educación. Recuperado el 20 de abril de 2013 desde www.mineduc.cl

MINEDUC (2010a). Manual proceso de seguimiento a la ejecución de acciones del Plan de Mejoramiento Educativo y evaluación de los compromisos del sostenedor. Santiago: Ministerio de Educación. Recuperado el 14 de mayo de 2012 desde www.comunidadescolar.cl

MINEDUC (2010b). Proceso de seguimiento a la ejecución de acciones del Plan de Mejoramiento Educativo y evaluación de los compromisos del sostenedor en los establecimientos 
Estudios Pedagógicos XLI, N 1: 237-256, 2015

TEORÍAS SUBJETIVAS DE DOCENTES DIRECTIVOS SOBRE FACTORES QUE DEFINEN LA EFICACIA DE LOS PLANES DE MEJORAMIENTO EDUCATIVO LEY SEP

adscritos a la Ley SEP. Santiago: Ministerio de Educación. Recuperado el 14 de mayo de 2012 desde www.comunidadescolar.cl

MINEDUC (2008a). Orientaciones para la elaboración del Plan de Mejoramiento Educativo. Santiago: Ministerio de Educación. Recuperado el 5 de mayo de 2012 desde www.planesdemejora.cl MINEDUC (2008b). Instructivo sobre metas de efectividad. Santiago: Ministerio de Educación. Recuperado el 5 de mayo de 2012 desde www.planesdemejora.cl

MINEDUC (2008c). Orientaciones para elaborar un sistema de monitoreo y seguimiento del Plan de Mejoramiento Educativo-Ley de Subvención Escolar Preferencial. Santiago: Ministerio de Educación. Recuperado el 5 de mayo de 2012 desde www.planesdemejora.cl

MINEDUC (2008d). Manual para la elaboración del Plan de Mejoramiento Educativo. Santiago: Ministerio de Educación. Recuperado el 5 de mayo de 2012 desde www.planesdemejora.cl

MINEDUC (s/f). Guía para el diagnóstico institucional de escuelas regulares con NT1 y/o NT2. Santiago: Ministerio de Educación. Recuperado el 14 de mayo de 2012 desde www.planesdemejora.cl

Muhr, T. (2003-2008). ATLAS.ti. Visual Qualitative Data Analysis (Version 5). Berlin: Scientific Software Development.

Murillo, F. J. (2003). La investigación sobre eficacia escolar en Ibero América. Revisión internacional sobre el estado del arte. Bogotá: Convenio Andrés Bello. Recuperado el 1 de agosto de 2011 desde http://www.uam.es/personal_pdi/stmaria/jmurillo/documentos/IIEE.pdf

Murillo, J. (2003). Una panorámica de la investigación Iberoamericana sobre eficacia escolar. Revista Electrónica Iberoamericana sobre Calidad, Eficacia y Cambio en Educación, vol.1 (1). Recuperado el 11 de agosto de 2011 desde http://www.inacap.cl/tportal/portales/tp4964b0e1bk102/ uploadImg/File/TeoriaPractEns/PracticasEns/6_EfectividadEducativa.pdf

Raczynski, D. y Muñoz, G. (2005). Efectividad escolar y cambio educativo en contextos de pobreza en Chile. Santiago: Ministerio de Educación. Recuperado el 22 de octubre de 2012 desde http://asesoriasparaeldesarrollo.cl/secciones/documentos/efectividad_escolar/RaczynskiMu\%C3\%B1 oz\%20Parte\%20I\%20Efectividad\%20Escolar\%20y\%20Cambio\%20Educativo\%20 en\%20Contextos\%20de\%20Pobreza\%20en\%20Chile.pdf

Strauss, A. y Corbin, J. (2002). Bases de la investigación cualitativa. Técnicas y procedimientos para desarrollar la teoría fundamentada. Medellín: Ediciones Universidad de Antioquia.

Taylor, S. y Bogdan, R. (1996). Introducción a los métodos cualitativos de investigación. Barcelona: Paidós.

Treviño, E., Órdenes, M. y Treviño, K. (2009). ¿Cómo el Plan de Mejoramiento de la Ley SEP puede ayudar a mejorar los aprendizajes? En Foco Educación, (2), 1-30. Recuperado el 26 de abril de 2012 desde http://www.cpce.cl/publicaciones/serie-en-foco-educacion/30-Ley-subvencion-escolarpreferencial

Valles, M. (2003). Técnicas cualitativas de investigación social. Reflexión metodológica y práctica profesional. Madrid: Síntesis.

Varguillas, C. (2006). El uso del Atlas Ti y la creatividad del investigador en el análisis cualitativo de contenido UPEL. Instituto Pedagógico Rural El Mácaro, Larus. Revista de Educación Universidad Pedagógica Experimental, vol.12 (12), 73-87. Recuperado el 17 de diciembre de 2012 desde http:// www.uam.es/personal_pdi/stmaria/jmurillo/Met_Inves_Avan/Materiales/Varguillas.pdf

Volante, P. y Müller, M. (2006). Cambios en el discurso sobre resultados de aprendizaje en escuelas de bajo rendimiento y alta vulnerabilidad social. Revista Pensamiento Educativo, vol.39 (2), 205-224. 